\title{
Leading the rebirth of the rural obstetrician
}

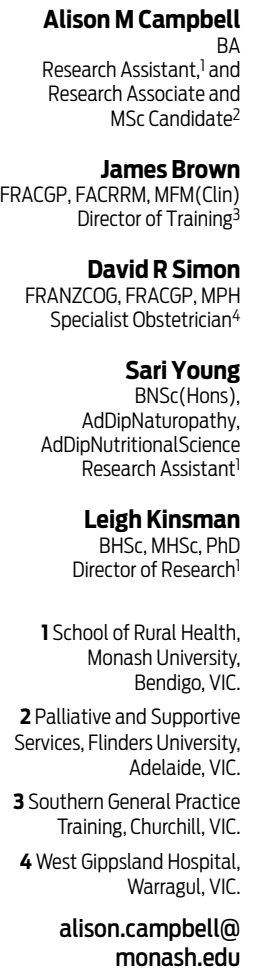

MJA 2014; 201: 667-670 doi: 10.5694/mjal4.00278

Editorial p 626 n 2002, 30\% of all Australian births occurred in non-metropolitan hospitals, and $57 \%$ of these hospitals did not provide specialist obstetric cover. ${ }^{1}$ Antenatal care led by general practitioner obstetricians is offered in $50 \%$ of South Australian and Victorian public hospitals and is the only public sector model in most nonmetropolitan hospitals. ${ }^{2}$ GP obstetric care has been shown to provide safe care for pregnant women at low risk of complications, and access to such services in rural Australia is essential. $3-8$

A looming crisis in the provision of rural obstetric services in Australia was identified in 2007.9 An important study of survey data from 2003 reported that Victorian GPs were becoming less likely to provide obstetric management and that half of the existing GP obstetricians intended to cease practising in the next 5-7 years. In addition, they found that $71 \%$ of GPs who completed a Diploma of the Royal Australian and New Zealand College of Obstetricians and Gynaecologists (DRANZCOG) did not then go on to practise independent procedural general practice obstetrics. ${ }^{9}$

Factors contributing to the forecast deficit in GP obstetric services included a rise in specialisation, centralisation of services, concerns regarding indemnity and litigation, rural workload and difficulty maintaining competence.,10-13 The problem of maintaining competence in rural environments has been compounded by reported difficulties in accessing appropriate locum coverage to allow attendance at upskilling courses, in addition to the time and travel required to participate. ${ }^{10}$

The impending shortage of GP obstetricians and the need for strategies to train, retrain and retain GP obstetricians in rural practice have been the integral considerations in developing a comprehensive training and support program offered in the Gippsland region of rural Victoria. The Gippsland region lies east of Melbourne, covering a land mass of 41524 square kilometres, and has a

\section{Abstrac}

Objectives: To understand the factors influencing the decisions of rural general practitioners and GP registrars to practise obstetrics, and to understand the impact on these decisions of an innovative obstetric training and support program in the Gippsland region of Victoria.

Design, setting and participants: Qualitative approach using semistructured interviews conducted in July and August 2013 and inductive content analysis. Participants were identified from training records over the previous 5 years for the Gippsland GP obstetric training and support program. Two questions were posed during interviews: What challenges face rural GPs in practising obstetrics? What impact has the Gippsland GP obstetric program had on GP obstetric career decisions?

Results: Of 60 people invited to participate, 22 agreed. Interviews ranged in duration from 40 to 90 minutes. The major themes that emerged on the challenges facing rural GPs in practising obstetrics were isolation, work-life balance and safety. The major themes that emerged on the impact of the Gippsland GP obstetric program were professional support, structured training and effective leadership.

Conclusion: Rural GP obstetricians are challenged by isolation, the impact of their job on work-life balance, and safety. The support, training and leadership offered by the Gippsland expanded obstetric training program helped doctors to deal with these challenges. The Gippsland model of training offers a template for GP obstetric procedural training programs for other rural settings.

population of around $240000 . .^{14}$ The program by Southern GP Training (SGPT) combines training for registrars and upskilling of GP obstetricians with strategies aimed at overcoming the professional isolation confronting rural GP obstetricians. The program (outlined in the Appendix; online at mja.com.au) expanded registrar training at the larger regional (specialist-led) units to include a 3-month rotation on secondment to a metropolitan hospital. Further training was extended to include a state government-funded 6- or 12-month placement in a GP-led obstetric practice (bridging post) with secondments to larger centres; provision of a clear, individualised postdiploma pathway with supported placement in a GP-led, communitybased obstetric practice; continued professional development; upskilling of existing GP obstetricians through the DRANZCOG Advanced qualification, which includes competence in performing caesarean sections; regular GP obstetrician meeting days attended by both registrars and practising GP obstetricians; specialist-led support and mentoring through a regular email forum; and specialist involvement in subregional GP perinatal education and morbidity meetings. In this way, the model provides a supported transition from specialist-led hospital obstetric units to GP-led, community-based obstetric services and integrates this with support for practising GP obstetricians.

The program is continuing to evolve, with new developments such as rotations to the Northern Territory and Pacific islands, ${ }^{15}$ to enrich the experience of the trainees. The implementation of this program has been matched by a period of recovery for Gippsland maternity services with an increase from $31 \mathrm{GP}$ obstetricians in 2007 to 39 in 2013, including an increase from 10 to 23 conducting caesarean sections. This represents a reversal of the pre-existing trend in service closures.9,16 Of the 39 currently practising GP obstetricians, 18 received their training in the SGPT Gippsland obstetric training program. ${ }^{17}$ Another three trainees went on to practise GP obstetrics elsewhere, meaning that $21 / 33$ program graduands were active in procedural practice. ${ }^{17}$

Recent government initiatives have supported GP obstetricians through funding professional development, incentive payments for upskilling, 
annual incentives for continuing GP obstetric practice and indemnity insurance support. These developments have removed some of the structural disincentives identified as barriers to procedural obstetric practice.

The aims of our study were to understand the factors influencing the decisions of rural GPs and GP registrars to practise obstetrics, and to understand the impact of this innovative GP obstetric training and support program on these decisions.

\section{Methods}

Our research was conducted in Gippsland in July and August 2013. Within the region, there are three specialist regional centres that offer a GP-led model of obstetrics, and five hospitals with GP-led services only, all with the facilities for caesarean sections.

Participants were identified from training records and the GP database of the past 5 years for the SGPT GP obstetrician and registrar training and support program. Letters of invitation, explanatory statements and consent forms were sent to potential participants.

We adopted a qualitative approach using semistructured face-to-face interviews. ${ }^{18-20}$ The research questions examined were:

- What challenges face rural GPs in practising obstetrics?

- What impact has the Gippsland GP obstetric program had on GP obstetric career decisions?

A three-stage framework method of data analysis (data display, data reduction and data interpretation) was applied, ${ }^{21}$ and measures were employed to augment the validity and reliability of this research. To ensure correct and detailed collection of participants' experience and views, all interviews were audiotaped, and copies of the transcripts were provided to participants to check for accuracy. Recorded interviews were analysed by two researchers for credibility and validation of the analysis. Analysis of the transcripts, once uploaded into NVivo 10 (QSR International), was conducted independently by two researchers to check interrater reliability of the emerging themes.
Ethics approval was obtained from the Monash University Human Research Ethics Committee for this research.

\section{Results}

Of the 60 potential participants contacted, 22 agreed to take part. The sample included registrars, GPs who were upskilling and established GP obstetricians who supported registrars in training. Interviews ranged from 40 to 90 minutes in duration.

Six major themes emerged: isolation, work-life balance, safety, professional support, structured training pathway and effective leadership.

The first three themes relate particularly to the first research question.

The theme of isolation included the subthemes of distance from specialist services, access to assistance, and access to professional development. The challenge of isolation came with the awareness that it was critical to have the confidence and competence to handle difficult situations and that access to assistance and advice was important. When experienced GPs talked about the impact of isolation, their comments were focused on managing a situation, often in the context of access to assistance from a local team.

\section{Neonatal Emergency Transfer Service (NETS) can come down, [but due to] the weather, it may be several hours before they can ... the GPs rally around and can keep working on the babies, in- tubate them, and keep breath- ing for them. It is not ideal, but it works well most times. (Participant $t$ )}

Comments about isolation from registrars and GPs who were at an earlier career stage focused on how access to assistance with the guidance and information available through the SGPT program ameliorated this isolation.

I've got someone to call on at the drop of a hat if I am out of my depth at any point, even if it's just for advice over the phone. (Participant e)

The theme of work-life balance included the subthemes of impact of after-hours call out, the demands of emergency situations, dealing with scheduled patients at the clinic after being at deliveries during the night, and family commitments.

Obstetrics interrupts the rest of life, both clinical, family life, and sleep. You know to be woken up in the middle of the night ... isn't a particularly pleasant thing, and try getting back to sleep after all the excitement. (Participant g)

Being part of a team of GP obstetricians assisted in achieving an acceptable work-life balance.

The theme of safety was mentioned more often by doctors who were at an early point on their career trajectory. This theme included the subthemes of patient safety and practitioner safety. Patient safety was related to backup and competence, while practitioner safety was about feeling supported and having confidence in dealing with the unknown. The SGPT Gippsland program was seen to contribute to improving safety.

Because (obstetrics) is a highrisk area and people burn out. They [SGPT] don't want us having disastrous situations when we are junior. (Participant a)

The second three themes - professional support, structured training pathway and effective leadership relate particularly to the second research question. Professional support was mentioned by all 22 participants. Participants from all groups within the cohort commented on the quality and availability of professional support within the Gippsland program. This theme included the subthemes of professional backup, professional networks and a respectful learning culture. With regard to professional backup, the availability of backup from specialists was described as timely and appropriate, as nominated mentors assisted with advice on practice in the clinic, and teams were built to support the training experience. Doctors in training and doctors in independent practice perceived they were well supported professionally.

When you are training you are always first on call, which is fantastic because you have to deal with everything that walks in the door. But you are paired with a consultant on the 
day. You basically run your assessment with them and see if they are happy with your plan, and for any instrumental deliveries or complicated issues you contact them to come in. So, it is very well supported. (Participant d)

Involvement in the Gippsland program made available both formal and informal professional networks to participants. The professional networks provided an environment where people at all stages of their career received support and timely, up-to-date information. Regular professional development opportunities were a valuable component, strengthening these networks and providing opportunities to reflect on best practice.

Ongoing professional development offered is fantastic, as it keeps you abreast of new developments as well as provides an opportunity for professional networking. (Participant $v$ )

A respectful learning culture with an emphasis on empowering and enabling participants was an important component of professional support.

Respect is a huge factor; the leaders in the program lead by example and are very inclusive and respectful of individuals' experience and needs. (Participant $u$ )

The structured training pathway theme emerged as an important component of the Gippsland GP obstetric program. This included the subthemes of community-based bridging posts for registrars; secondment for additional experience; and continuous professional development. Registrars rated the bridging posts as critical to offering a safe transition.

I think it is about fostering supported practice and this is a particular time of vulnerability in terms of support ... the movement from hospital-based practice to being a new person in community-based practice. (Participant g)

The theme of effective leadership was apparent across all interviews. There was clearly the perception of supportive, knowledgeable and respectful leadership within the program, and this was highly valued.

They are definitely good mentors and good role models and that is part of the reason ... to want to keep going with this pathway. (Participant $f$ )

\section{Discussion}

The themes of isolation, work-life balance and safety for the practitioners and patients emerged in our study as substantial challenges for rural GPs in practising obstetrics. These findings are consistent with the findings of other researchers who have studied the challenges of rural and remote medical practice more broadly. ${ }^{22,23}$ Work-life balance is particularly important for sustainable practice ${ }^{24}$ and is vulnerable to the demands of isolated obstetric practice. Our study indicates that the Gippsland GP obstetric program has contributed to a recovery and retention of maternity units in Gippsland founded on its success in helping doctors deal with these challenges.

Participants found the obstetric program to be professionally supportive, with meaningful backup, advice and support of professional development. The program has also been instrumental in building and supporting professional networks. Reliable, relevant backup and advice ameliorates isolation and enhances patient and practitioner safety. Professional networks remove isolation and enable cooperative rostering, which is a means to improving work-life balance. In this way, the SGPT Gippsland GP obstetric program would seem to have become fundamental for sustaining GP obstetric practice in Gippsland.

Our study suggests that the structure of the Gippsland GP obstetric training enables its trainees to continue into active, independent procedural obstetric practice. The bridging post after the primary training was highly valued. A large decrease in use of procedural skills 1 year after their primary procedural training has been reported previously. ${ }^{9,25}$ Supported transition after completion of hospital-based training has been found to be an important factor influencing recently qualified GPs to continue into independent procedural practice. ${ }^{25}$ Structured, respectful clinical supervision by senior role models is vital to effective postgraduate medical education, ${ }^{26}$ with the supervision relationship being shown to be more important than the supervision method. ${ }^{27}$

Leadership was clearly a major factor in the impact and success of the SGPT Gippsland GP obstetric program. This leadership was provided by committed specialist obstetricians and active GP obstetricians.

There was a notable absence in the data of mention of financial disincentives to practising GP obstetrics. This suggests that disincentives identified previously ${ }^{1}$ have been largely removed by recent government initiatives in this area.

This study was conducted in a particular geographic area, so transferability of the results cannot be assumed. In particular, this program was introduced where a shortage of GP obstetricians was forecast but not yet apparent. The participation of GP obstetricians was key to the success of the program. Therefore, this program design may not be as effective where GP obstetrician shortages already exist. However, themes such as isolation, safety and leadership are likely to be relevant in most rural settings, and the strength of these themes across the different practitioner groupings and towns suggests that the findings are generalisable. The stratified sampling method used was a strength of the study.

Our study also suggests that the Gippsland GP obstetric program has had a substantial impact on trainees continuing into active obstetric practice and on GP obstetricians continuing in their obstetric practice. This innovative program was made possible by state and federal government funding, the support of local and metropolitan hospitals, and ownership by both specialist and GP obstetricians. Leadership, organisational support and administrative support by SGPT have provided the scaffolding for the program. Key features of this training include a supported transition into community-based GP obstetrics; adequate clinical exposure through secondments; a culture supportive of GP obstetrics; building and 
sustaining professional support networks; and inspirational leadership. The increase in numbers of practising GP obstetricians has enabled more acceptable rosters and greater flexibility in accommodating personal commitments. These key features should be foundational considerations in replicating this successful model elsewhere.

Acknowledgements: We acknowledge the Victorian Department of Health, Health Workforce Division, for their funding of the GP obstetrician training program, and the clinicians at Western Hospital and Southern Health Clayton and Dandenong for providing valuable clinical placements for Gippsland registrars and GPs.

Competing interests: No relevant disclosures.

Received 28 Feb 2014, accepted 29 Sep 2014.

1 Robson S, Bland P, Bunting M. An anonymous survey of provincial, rural and remote obstetricians' long-term practice intentions; implications for the provision of specialist obstetric services outside metropolitan areas in Australia. Aust N Z J Obstet Gynaecol 2005; 45: 395-398.

2 Sutherland G, Yelland J, Wiebe J, et al. Role of general practitioners in primary maternity care in South Australia and Victoria. Aust NZ J Obstet Gynaecol 2009; 49: 637-641.

3 Kirke AB. How safe is GP obstetrics? An assessment of antenatal risk factors and perinatal outcomes in one rural practice. Rural Remote Health 2010; 10: 1545.

4 Pesce AF. Rural maternity units: how will they have a future [editorial]? Med J Aust 2008; 188: 70-71.
5 Cameron B, Cameron S. Outcomes in rural obstetrics, Atherton Hospital 1991-2000. Aust J Rural Health 2001; 9 Suppl 1: S39-S42.

6 Hemminki E, Heino A, Gissler M. Should births be centralised in higher level hospitals? Experiences from regionalised health care in Finland. BJOG 2011; 118: 1186-1195.

7 Tracy SK, Sullivan E, Dahlen H, et al. Does size matter? A population-based study of birth in lower volume maternity hospitals for low risk women. BJOG 2006; 113: 86-96.

8 Woollard LA, Hays RB. Rural obstetrics in NSW. Aust N Z J Obstet Gynaecol 1993; 33: 240-242.

9 Loy CS, Warton RB, Dunbar JA. Workforce trends in specialist and GP obstetric practice in Victoria. Med J Aust 2007; 186: 26-30.

10 Koppula S, Brown JB, Jordan JM. Experiences of family medicine residents in primary care obstetrics training. Fam Med 2012; 44: 178-182.

11 Robinson M, Slaney GM, Jones GI, Robinson JB. GP Proceduralists: 'the hidden heart' of rural and regional health in Australia. Rural Remote Health 2010; 10: 1402.

12 Wiegers TA. General practitioners and their role in maternity care. Health Policy 2003; 66: 51-59.

13 Watts RW, Marley JE, Beilby JJ, et al. Training, skills and approach to high-risk obstetrics in rural GP obstetricians. Aust N Z J Obstet Gynaecol 1997; 37: 424-426.

14 Australian Bureau of Statistics. National Regional Profile: Gippsland (Statistical Division). Canberra: ABS, 2010. http://www. abs.gov.au/AUSSTATS/abs@.nsf/78leb7868ce e03e9ca2571800082bece/1532el6036381be2 ca25771300180e73!OpenDocument (accessed Jul 2014).

15 Sterling L. Post-DRANZCOG pathway. O\&G Magazine 2013: 15: 42-43. http://www.ranzcog. edu.au/editions/doc_view/1500-42-postdranzcog-pathway.html (accessed Sep 2014).

16 Rural Doctors Association of Victoria. Victorian rural procedural GP workforce and age statistics 04-09. http://www.rdav.com.au/ Uploads/Documents/2009SurveyGP\%20 Proceduralists_20140811111345.pdf (accessed Nov 2014).

17 Brown J, Simon D, Young S, Kinsman L. Leading the rebirth of the rural obstetrician. Presentation at General Practice Education and Training Convention; 2013 Sep 11-12; Perth, Australia.

18 Sandelowski M. Whatever happened to qualitative description? Res Nurs Health 2000; 23: 334-340.

19 Munhall P. Qualitative research. In: Brink PJ, Wood MJ, editors. Advanced design in nursing research. 2nd ed. London: Sage, 1998: 335-355.

20 Endacott R. Clinical research 4: qualitative data collection and analysis. Intensive Crit Care Nurs 2005; 21: 123-127.

21 Miles M, Huberman M. Qualitative data analysis. Thousand Oaks, Calif: Sage, 1984.

22 Henry JA, Edwards BJ, Crotty B. Why do medical graduates choose rural careers? Rural Remote Health 2009; 9: 1083.

23 Wakerman J, Humphreys JS, Wells R, et al. Features of effective primary health care models in rural and remote Australia: a casestudy analysis. Med J Aust 2009; 191: 88-91.

24 Russell DJ, McGrail MR, Humphreys JS, Wakerman J. What factors contribute most to the retention of general practitioners in rural and remote areas? Aust J Prim Health 2012; 18 : 289-294.

25 McKenzie A, Beaton N, Hollins J, et al. Advanced rural skills training: are recently qualified GPs using their procedural skills? Rural Remote Health 2013; 13: 2159.

26 Hore CT, Lancashire W, Fassett RG. Clinical supervision by consultants in teaching hospitals. Med J Aust 2009; 191: 220-222.

27 Kilminster SM, Jolly BC. Effective supervision in clinical practice settings: a literature review. Med Educ 2000; 34: 827-840. 